\title{
STRUCTURE OF PARTICLE-LADEN TURBULENT WATER JETS IN STILL WATER
}

\author{
R. N. Parthasarathy and G. M. Faeth ${ }^{\dagger}$ \\ Department of Aerospace Engineering, University of Michigan, Ann Arbor, MI 48109-2140, U.S.A.
}

(Received 10 February 1987; in revised form 17 May 1987)

\begin{abstract}
The structure of turbulent, dilute, particle-laden water jets, submerged in still water, was studied both experimentally and theoretically. Nonintrusive measurements were made of mean and fluctuating phase velocities and particle number fluxes. Analysis was used to help interpret the measurements. considering three limiting cases, as follows: (1) locally-homogeneous flow, where relative velocities between the phases are ignored; (2) deterministic separated flow, where relative velocities are considered, but particle/turbulence interactions are ignored; and (3) stochastic separated flow, where both phenomena are considered using random-walk methods. The locally-homogeneous flow approximation was more effective than for past work involving larger density ratios between the phases; however, stochastic analysis yielded best agreement with measurements. Effects of enhanced drag (due to high relative turbulent intensities of particle motion) and effects of particles on liquid turbulence properties (turbulence modulation), were observed. Several recent proposals for treating these phenomena were examined; however, none appears to be adequate for reliable general use.
\end{abstract}

\section{INTRODUCTION}

Particle-laden turbulent jets in liquids are encountered in industrial and natural processes. Furthermore, these flows provide a useful simulation of sprays in high-pressure environments, since they have comparable phase-density ratios. The present study considers particle-laden turbulent water jets in still water, motivated by these applications. The work involved both measurements of flow structure and analysis to help interpret the measurements and to initiate development of predictive methods for these flows. The study was limited to nearly monodisperse particles and dilute particle concentrations (particle volume fractions $<5 \%$ ).

A complete discussion of earlier studies of particle-laden jets will not be undertaken since reviews of these flows have recently appeared (Faeth 1983, 1987). The present study extends earlier work on multiphase jets in this laboratory, which included: particles in gases (Shuen et al. 1983a,b, 1985); drops and sprays in gases (Solomon et al. 1985a,b; Shuen et al., 1986); and bubbles in liquids (Sun \& Faeth 1986; Sun et al. 1986). The earlier flows involved either very large or very small phase-density ratios, yielding different responses of the dispersed phase to the motion of the continuous phase. However, conditions where phase densities are comparable were not considered; therefore, the present study was designed to help fill this gap in the literature. Comparable phase densities are of particular interest, since all effects of interphase momentum transfer are important-particle inertia, virtual mass, drag and the Basset history force. Furthermore, these flows also exhibit high relative turbulence intensities for particle motion, which influence particle drag properties (Clift et al. 1978). Thus, they represent a good test of methods used to predict particle motion in turbulent environments.

The present experiments involved solid glass spheres (roughly $0.5 \mathrm{~mm} \mathrm{dia}$ ) in water, yielding a phase-density ratio of $2.45: 1$. Mean and fluctuating phase velocities were measured using a phase-discriminating laser-Doppler anemometer (LDA). Particle number fluxes were measured by detecting Mie scattering from particles within a laser light sheet. As a baseline, measurements of flow properties in a pure water jet were completed, using the same injector. Particle drag properties were calibrated, using separate single-particle experiments, in order to reduce uncertainties in separated-flow analysis.

Analysis of flow properties was similar to recent work in this laboratory (Shuen et al. 1983a,b, 1985; Solomon et al. 1985a,b; Sun \& Faeth 1986; Sun et al. 1986). Three limiting approximations

tTo whom all correspondence should be addressed. 
were considered, as follows: (1) locally-homogeneous flow (LHF), where interphase transport rates are assumed to be infinitely fast so that the relative velocities of the phases can be ignored; (2) deterministic separated flow (DSF). where the relative velocities between the phases are considered. but particle turbulence interactions are ignored; and (3) stochastic separated flow (SSF), where effects of both relative velocities and particle/turbulence interactions are considered using random sampling for turbulence properties, in conjunction with random-walk computations for particle motion. Exact numerical simulation of multiphase turbulent jets is not feasible for Reynolds numbers encountered in practice, due to the large range of length scales in the flow. Thus, continuous-phase turbulence properties were analyzed using widely adopted methods of $k-\epsilon$ turbulence models, while averaging over phenomena on the scale of particle size, similar to past treatments of multiphase jets in this laboratory. All empirical aspects of the turbulence model, however, were established by early work with constant density single-phase shear flows (Lockwood \& Naguib 1975) and subsequently verified by comparison with measurements in constant- and variable-density single-phase round jets (Jeng \& Faeth 1984). Two extensions of the analysis were also undertaken, as follows: (1) consideration of effects of anisotropy of continuous-phase turbulence on the anisotropy of fluctuating particle motion, since Shuen et al. (19.85) suggested that this phenomenon was important; and (2) consideration of effects of particles on the turbulence structure of the continuous phase, called turbulence modulation by Al Taweel \& Landau (1977).

The paper begins with a description of theoretical and experimental methods. This is followed by discussion of the particle drag calibration, measurements of near-injector flow properties to establish initial conditions for structure computations, and baseline results for single-phase water jets in still water. The paper concludes with a description of structure measurements in particle-laden jets and their comparison with predictions.

\section{EXPERIMENTAL METHODS}

\subsection{Apparatus}

The flow was observed within a windowed test tank $(410 \times 530 \times 910 \mathrm{~mm}$ high $)$ used during earlier studies of bubbly jets (Sun \& Faeth 1986; Sun et al. 1986) The injector was a constant-area passage $(5.08 \mathrm{~mm}$ dia, $350 \mathrm{~mm}$ long) injecting vertically downward. This arrangement yielded nearly fully-developed pipe flow at the exit, for single-phase flow conditions. Instrumentation was mounted rigidly; therefore, the injector was traversed in three directions to measure flow properties. Positioning accuracies were $100 \mu \mathrm{m}$ in the horizontal plane and $500 \mu \mathrm{m}$ in the vertical direction.

Filtered water was supplied to the injector by a rotary gear pump. A valve, bypass and surge tank in the pump exhaust line were used to control and smooth the flow. Water flow rates were measured with a rotameter, which was calibrated by collecting water for timed intervals. Water injected into the test tank was removed by an overflow pipe and returned to a reservoir at the pump inlet.

Dried particles were fed by a screw feeder (maximum feed rate variations of $4 \%$ ) to a standpipe located directly above the injector. While falling under the the influence of gravity, the particles mixed with the water and entered the injector flow at a tee. After passing through the jet, the particles collected naturally at the bottom of the tank, where they were removed periodically by a suction system.

\subsection{Instrumentation}

Particle velocities. Mean and fluctuating particle velocities were measured with an LDA. A dual-beam arrangement was used, based on the $514.5 \mathrm{~nm}$ line of an $\mathrm{Ar}^{+}$laser operated at $200 \mathrm{~mW}$ optical power. Off-axis $\left(45^{\circ}\right)$ forward-scatter light detection was used for particle velocity measurements in order to control the size of the measuring volume. Directional bias and ambiguity were eliminated by use of a $40 \mathrm{MHz}$ Bragg cell frequency shifter. Output signals were downshifted to convenient frequency ranges for filtering and signal processing.

Signal amplitudes from the naturally-seeded water were much smaller than from particles; therefore, particle signals were identified by reducing the gain of the detector circuit until only signals from particles were recorded. This procedure was verified by stopping the flow of particles, 
which invariably resulted in no further signals being processed. Particle velocities were found using a burst-counter signal processor (TSI Model 1990C). The output of the burst counter was stored and subsequently processed by a microcomputer (IBM 9002) to yield particle-averaged mean and fluctuating velocities. Predictions of particle velocities were averaged in the same way, for comparison with the measurements. Streamwise and radial particle velocities were measured by appropriately orienting the optical plane of the LDA.

The LDA measuring volume for particle velocities was relatively large $(0.6 \mathrm{~mm}$ dia, $0.7 \mathrm{~mm}$ long) since grazing collisions of particles with the measuring volume were recorded. Nevertheless, gradient broadening was small and uncertainties of these measurements largely resulted from finite sampling times. Estimates of experimental uncertainties $(95 \%$ confidence) are as follows: mean streamwise velocities, 5\%: fluctuating streamwise and radial velocities, $10 \%$; and mean radial velocities, $50 \%$. Uncertainties of mean radial velocities are high due to their small magnitude.

Liquid velocities. Light scattered by natural seeding in the water yields lower-amplitude signals than light scattered by the glass beads; therefore, simple amplitude discrimination can help to distinguish between liquid-phase and particle velocity signals. As Modarress et al. (1984) point out, however, particles grazing the LDA measuring volume also yield low-amplitude signals, which can be interpreted as coming from the liquid phase, biasing the liquid velocity measurements.

A phase-discriminator system, similar to that used by Modarress et al. (1984), was used to avoid biasing from grazing particle collisions with the LDA measuring volume. The basic LDA arrangement was similar to the particle velocity measurements, but with on-axis forward-scatter light detection. The phase discriminator involved a third beam, from a $5 \mathrm{~mW} \mathrm{HeNe}$ laser $\left(18^{\circ}\right.$ from the LDA axis) which was focused at the LDA measuring volume and observed by off-axis $\left(32^{\circ}\right)$ detection through a laser-line filter. The discriminator optics were adjusted so that all grazing collisions were observed, i.e. the region viewed $(0.6 \mathrm{~mm}$ dia, $1.2 \mathrm{~mm}$ long) had a diameter greater than the sum of the diameters of the LDA measuring volume and the largest particles. The discriminator signal was recorded simultaneously with the velocity signal from the burst counter. The data-processing system was programmed to eliminate all velocity records where a pulse on the discriminator signal indicated the presence of a particle. The time between valid liquid velocity measurements was small in comparison to integral time scales of the flow; therefore, the velocity signal was time averaged, ignoring periods when particles were present, to obtain unbiased time-averaged mean and fluctuating liquid velocities. Natural seeding in the water was sufficient to yield high data rates $(3-8 \mathrm{kHz})$. Various velocity components and the Reynolds stress were obtained by rotating the optical plane of the LDA, as described by Durst \& Whitelaw (1971).

The LDA measuring volume for liquid velocities was $0.1 \mathrm{~mm}$ dia $\times 1.3 \mathrm{~mm}$ long. Gradient broadening was negligible and experimental uncertainties were dominated by finite sampling times. Estimated experimental uncertainties ( $95 \%$ confidence) are as follows: mean streamwise velocities, $5 \%$; fluctuating streamwise and radial velocities, $10 \%$; turbulence kinetic energy, $20 \%$; and Reynolds stress, $30 \%$ at its maximum and proportionately higher elsewhere.

Particle number fluxes. Mie scattering was used to measure particle number fluxes in the streamwise direction. A small light sheet, having nearly-uniform intensity, was produced at the measuring volume by passing the beam from a $5 \mathrm{~mW}$ HeNe laser through an aperture. The measuring volume was observed in the horizontal plane, normal to the laser beam. Particles passing through the measuring volume generated pulses in the detector output. The pulses were shaped and recorded by a pulse counter which had an adjustable threshold to control spurious background signals. Grazing collisions of particles with the optical measuring volume were recorded; therefore, the radius of the region observed was roughly the sum of the optical radius and the particle radius (total size roughly $0.75 \times 0.75 \mathrm{~mm}$ ). The actual area of observation, however, was calibrated by collecting particles in a uniform flow. In general, more than 1000 particles were counted in order to find the mean particle number flux.

Experimental uncertainties for the particle number flux measurements were due to variable particle diameters, which influences the area actually observed; gradient broadening; and finite sampling times. The latter dominated the measurements, yielding uncertainties $(95 \%$ confidence) of $<15 \%$ along the axis and proportionately higher elsewhere. 
Table 2. Source terms and empirical constants in separated-flow analysis ${ }^{2}$

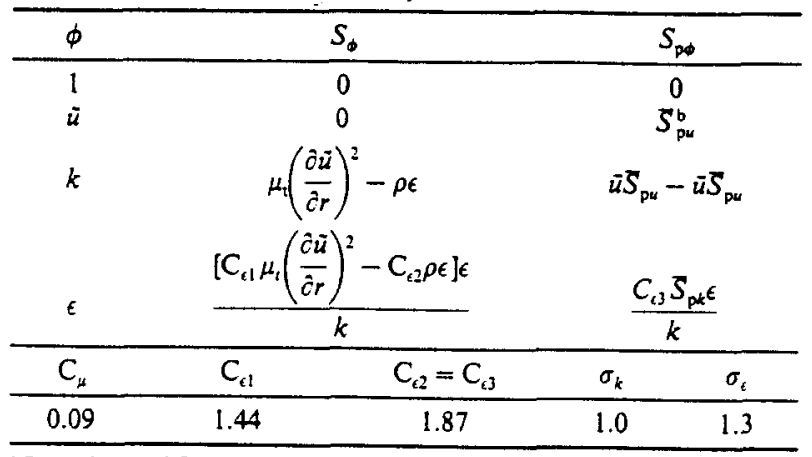

${ }^{2} S_{p k}$ only used for the SSF-KMOD and SSF-EXT versions; $S_{p c}$ only used for the SSF-EXT version.

$$
{ }^{b} \bar{S}_{\mathrm{pu}}=\frac{\sum_{i=1}^{n} \dot{n}_{\mathrm{i}}\left[m_{\mathrm{p}}\left(u_{\mathrm{p} \mathrm{in}}-u_{\mathrm{pout}}\right)+a\left(1-\frac{\rho}{\rho_{\mathrm{p}}}\right) \Delta t_{\mathrm{p}}\right]}{V_{j}} .
$$

We use an axisymmetric coordinate system with streamwise and radial distances, $x, r$ and velocities $u, v$. The streamwise direction is vertically downward, aligned with the gravitational acceleration vector which has a magnitude, $a$. With this arrangement, the general form of the governing equations is as follows:

$$
\frac{\partial}{\partial x}(\bar{\rho} \tilde{u} \phi)+r^{-1} \frac{\partial}{\partial r}(r \bar{\rho} \tilde{v} \phi)=r^{-1} \frac{\partial}{\partial r}\left(\frac{r \mu_{\mathrm{t}}}{\sigma_{\phi}} \frac{\partial \phi}{\partial r}\right)+S_{\phi}+S_{\mathrm{p} \phi},
$$

where

$$
\tilde{\phi}=\frac{\overline{\rho \phi}}{\bar{\rho}}
$$

denotes a Favre average, an overbar denotes a conventional time average, $\rho$ is the density, $\mu_{1}$ is the turbulent viscosity, $\sigma_{\phi}$ is a turbulent Prandtl-Schmidt number and $S_{\phi}$ and $S_{\mathrm{p} \phi}$ are source terms resulting from the liquid and particle phases, respectively. Governing equations are solved for conservation of mass $(\phi=1)$, conservation of momentum $(\phi=\tilde{u})$, turbulence kinetic energy $(\phi=k)$ and the rate of dissipation of turbulence kinetic energy $(\phi=\epsilon)$. The source terms, $S_{\phi}$ and $S_{\mathrm{p} \phi}$, are summarized in table 2 , along with the empirical constants, $\mathrm{C}_{\phi}$, used during the computations. The $S_{\phi}$ terms are conventional for a single-phase jet (Lockwood \& Naguib 1975); the particle source terms, $S_{\mathrm{p} \phi}$, will be discussed later. The turbulent viscosity was computed as usual:

$$
\mu_{\imath}=\frac{\mathrm{C}_{\mu} \rho k^{2}}{\epsilon}
$$

The flow leaving the injector was similar to fully-developed flow and had no potential core. Initial conditions for the computations were prescribed from measurements near the injector, as described later. Ambient values of $\dot{u}, k$ and $\epsilon$ are zero, while gradients of these quantities are zero at the axis of the flow, from symmetry.

\subsection{Dispersed Phase (SSF Formulation)}

The particle phase was treated by solving Lagrangian equations for the trajectories of a sample of individual particles ( $n$ groups defined by initial position, velocity, direction and sample) as they move through the flow and encounter a random distribution of turbulent eddies. Results of these computations were averaged over all particle groups, to provide mean and fluctuating particle properties as well as the particle source terms, $S_{\mathrm{p} \phi}$, needed to solve the continuous-phase governing equations. 
Several variations of the SSF analysis were considered, in order to examine effects of anisotropic turbulence properties and effects of particles on continuous-phase turbulence properties (turbulence modulation). The baseline version follows Shuen et al. (1983a,b, 1985), which is a modification and extension of a method proposed by Gosman \& Ioannides (1981). In all cases, properties are assumed to be uniform within each eddy and to change randomly from one eddy to the next. At the start of particle/eddy interaction, the velocity of the eddy is found by making a random selection from the probability-density function (PDF) of velocity. A particle is assumed to interact with an eddy for a time which is the minimum of either the eddy lifetime or the time required for the particle to cross the eddy. Characteristic eddy sizes, $L_{\mathrm{e}}$. and lifetimes, $t_{\mathrm{a}}$, are estimated from the following expressions (Shuen et al. 1983b, 1985):

$$
L_{\mathrm{e}}=\frac{\mathrm{C}_{\mu}^{3,4} k^{3.2}}{\epsilon}, \quad t_{\mathrm{e}}=\frac{L_{z}}{\left(\frac{2 k}{3}\right)^{1,2}} .
$$

Particles and eddies are assumed to interact as long as the time of interaction and the relative displacement of the particle and eddy are both less than $t_{\mathrm{e}}$ and $L_{\mathrm{e}}$.

During baseline SSF analysis, the velocity PDF was taken to be isotropic and Gaussian, having standard deviations

$$
\left(\frac{2 k}{3}\right)^{1 / 2}
$$

and mean values $\bar{u}, \bar{v}$ with the tangential mean velocity $\bar{w}=0$. However, the streamwise velocity fluctuations of particles in particle-laden jets are generally underestimated using this approach, an effect which has been attributed to ignoring the anisotropy of turbulent fluctuations generally observed in these flows (Shuen et al. 1985; Sun \& Faeth 1986). For the present initial considerations, anisotropy levels in the multiphase flows were correlated directly from the measurements and applied to the velocity PDFs, to minimize uncertainties in evaluation of the phenomenon.

Initial conditions for separated flow analysis were specified at $x / d=8$, which was the position nearest the injector where all needed measurements could be made with acceptable spatial resolution and accuracy. Downstream of this position, particle volume fractions were $<3 \%$ : therefore, particle collisions and effects of adjacent particles on particle transport properties are negligible (Faeth 1987). Particle dimensions were $<10 \%$ of the flow half-width; therefore, particles were assumed to be in a locally uniform environment, based on liquid properties at their center. Particles were assumed to be spherical, and Magnus and Saffman lift forces and static pressure gradients were neglected, similar to Shuen et al. (1983b).

Under these assumptions, particle motion was found using the formulation of Odar \& Hamilton (1964), reviewed by Clift et al. (1978), as follows:

$$
\frac{\mathrm{d} x_{\mathrm{p} i}}{\mathrm{~d} t}=u_{\mathrm{p} i}
$$

and

$$
\left(\frac{\rho_{\mathrm{p}}}{\rho}+\frac{\Delta_{\mathrm{A}}}{2}\right) \frac{\mathrm{d} u_{\mathrm{ri}}}{\mathrm{d} t}=a\left(\frac{\rho_{\mathrm{p}}}{\rho}-1\right) \delta_{t i}-3 C_{\mathrm{D}}\left|u_{\mathrm{r}}\right| \frac{u_{\mathrm{r} i}}{\left(4 d_{\mathrm{p}}\right)}-\Delta_{\mathrm{H}}\left(\frac{81 \nu}{\pi d_{\mathrm{p}}^{2}}\right)^{1 / 2} \int_{t_{0}}^{t}(t-\zeta)^{-1 / 2}\left(\frac{\mathrm{d} u_{\mathrm{ri}}}{\mathrm{d} \xi}\right) \mathrm{d} \xi,
$$

where $x_{\mathrm{pi}}, u_{\mathrm{p} i}$ and $u_{\mathrm{r} i}$ denote particle position and velocity, and the particle velocity relative to the liquid, in a Cartesian reference frame where $i=1$ (vertical), 2 and 3 . Other symbols appearing in [5] and [6] are defined as follows: $\rho_{\mathrm{p}}$ is the particle density, $d_{\mathrm{p}}$ is the particle diameter, $t$ and $t_{0}$ are the time and time at the start of interaction, $\Delta_{\mathrm{A}}$ and $\Delta_{\mathrm{H}}$ are parameters to account for particle acceleration, $\delta_{j i}$ is the Kronecker delta function, $C_{D}$ is the drag coefficient, $\left|u_{\mathrm{T}}\right|$ is the magnitude of the relative velocity vector, $v$ is the kinematic viscosity of the liquid and $\zeta$ is a dummy variable of integration. The terms on the 1.h.s. of [6] represent accelerations due to particle and virtual mass, while the terms on the r.h.s. represent buoyancy, drag and Basset history forces.

The parameters $\Delta_{A}$ and $\Delta_{H}$ were empirically correlated by Odar \& Hamilton (1964), as follows:

$$
\Delta_{A}=2.1-\frac{0.123 M_{\mathrm{A}}^{2}}{\left(1+0.12 M_{\mathrm{A}}^{2}\right)}
$$


and

$$
\Delta_{\mathrm{H}}=0.48+\frac{0.52 M_{\mathrm{A}}^{3}}{\left(1+M_{\mathrm{A}}\right)^{3}},
$$

where $M_{\mathrm{A}}$ is the particle acceleration modulus,

$$
M_{\mathrm{A}}=\left(\frac{\mathrm{d} u_{\mathrm{r}}}{\mathrm{d} t}\right) \frac{d_{\mathrm{p}}}{u_{\mathrm{r}}^{2}}
$$

The values of $\Delta_{\mathrm{A}}$ and $\Delta_{\mathrm{H}}$ vary in the ranges 1.0-2.1 and 1.00-0.48, the former values being the correct limit for the Basset-Boussinesq-Oseen (BBO) formulation of [6] (Clift et al. 1978).

For baseline computations, the BBO limit was used. Particle Reynolds numbers, based on the relative velocity of the particle and fluid, did not reach the supercritical flow regime; therefore, the standard drag coefficient for solid spheres was approximated as follows:

$$
C_{\mathrm{D}}=\frac{24\left(1+\frac{\operatorname{Re}^{2 / 3}}{6}\right)}{\operatorname{Re}}, \quad \operatorname{Re} \leqslant 1000 ; \quad C_{\mathrm{D}}=0.44, \quad \operatorname{Re}>1000 ;
$$

where $R e$ denotes the particle Reynolds number.

Computations revealed that turbulent fluctuations of the continuous phase were often large in comparison to relative velocities; therefore, the extended version of the SSF analysis accounted for this effect, following Clift et al. (1978) and Lopes \& Dukler (1986). The expression for the drag coefficient in this case is as follows:

$$
\begin{aligned}
& C_{\mathrm{D}}=\frac{162 I_{\mathrm{r}}^{1 / 3}}{\operatorname{Re}}, \quad \operatorname{Re} \leqslant 50 \\
& C_{\mathrm{D}}=0.133\left(1+\frac{150}{\operatorname{Re}}\right)^{1.565}+4 I_{\mathrm{r}}, \quad 50<\operatorname{Re}<700
\end{aligned}
$$

where

$$
I_{\mathrm{r}}=\frac{\left(\frac{2 k}{3}\right)^{1 / 2}}{u_{\mathrm{r}}}
$$

assuming isotropic turbulence. These expressions were developed for $I_{\mathrm{r}} \leqslant 0.5$; however, they were used during the present calculations for $I_{\mathrm{f}}$ ranging up to 1.5 , for lack of an alternative.

\subsection{Particle Source Terms (SSF Formulation)}

The interaction between the particle and liquid phases yields source terms, $S_{\mathrm{p \phi}}$ in the governing equations for the liquid phase. For the baseline SSF analysis, this interaction is limited to the particle source term in the mean momentum equation $\left(S_{\mathrm{pu}}\right.$ in table 2$)$, ignoring effects of turbulence modulation. In the expression for $S_{p u}$ in table $2, \dot{n}_{i}$ is the number of particles per unit time in group $i, m_{\mathrm{p}}$ is the particle mass, while "in" and "out" denote conditions entering and leaving computational cell $j$ (having a cell volume $V_{j}$ ). The flow rate of particles along a given trajectory is conserved; therefore, $\dot{n}_{i}$ is a constant specified near the injector to satisfy total particle flow-rate requirements. The last term in $S_{\mathrm{pu}}$ accounts for the effects of buoyancy on the flow; here $\Delta t_{\mathrm{p}}$ is the residence time of a particle in a computational cell (Sun \& Faeth 1986).

Two approaches were examined to treat the effects of turbulence modulation, denoted SSF-KMOD and SSF-EXT, similar to the limiting cases recently considered by Reitz \& Diwakar (1987). Both versions adopt the source term $S_{\mathrm{pk}}$ given in table 2, which is the same as in Shuen et al. (1985). This term can be computed exactly from the SSF analysis and does not require modeling. The analogous term in the $\epsilon$ equation has to be modeled. This term is simply ignored for the SSF-KMOD version, i.e. $S_{\mathrm{pc}}=0$. For the SSF-EXT approach, $S_{\mathrm{pc}}$ is modeled by assuming that it is proportional to the source term in the $k$ equation, similar to treatments of this term by Reitz \& Diwakar (1987) and in single-phase flows (Lockwood \& Naguib 1975). This introduces a new empirical constant $C_{t 3}$. The value of $C_{t 3}$ was chosen by considering equilibrium requirements 
in a homogeneous stationary flow where turbulence is only generated by particle motion; this implies that $\mathrm{C}_{63}=\mathrm{C}_{62}$.

\subsection{Numerical Solution}

Calculations for the liquid phase were performed using the GENMIX algorithm of Spalding (1977), which uses a second-order implicit central-difference scheme. The computational grid was similar to that in Shuen et al. (1983a,b 1985): 33 crossstream grid nodes, with streamwise step sizes limited to $6 \%$ of the current flow width or an entrained flow increase of $5 \%$-whichever was smaller. The particle phase was computed by a second-order finite-difference algorithm employing 7200 and 9600 trajectories for case I and II flows, respectively.

\subsection{Simplified Analysis}

DSF method. All turbulence/particle interactions are ignored using the DSF approach. Particle trajectories are found by integrating [5] and [6] with local mean liquid velocities replacing instantaneous eddy velocities. Thus, each initial condition yields a single deterministic trajectory and only $S_{\mathrm{pu}}$ is considered in the solution for liquid-phase properties. Particle drag was computed from [10], similar to the baseline SSF approach. The number of particle trajectories was also the same as for the SSF computations.

LHF method. This approximation implies that both phases have the same instantaneous velocity at each point; therefore, the flow corresponds to a variable-density single-phase flow whose density changes due to changes in particle concentration. Turbulent dispersion of particles is then equivalent to the turbulent diffusion of liquid, and particle inertia fully influences turbulence properties. Thus, the method allows for turbulence modulation, to the extent that negligible relative velocities between the phases is correct.

The formulation in this case is identical to that in Shuen et al. (1983a,b, 1985) and Sun and coworkers (Sun \& Faeth 1986; Sun et al. 1986). The procedure follows the conserved-scalar formalism which is widely used for flows having variable scalar properties (Bilger 1976; Faeth 1987).

\section{RESULTS AND DISCUSSION}

\subsection{Drag Calibrations}

Particle drag was calibrated by measuring the terminal velocities of single particles in still water. This was carried out by positioning a plate containing a small aperture below the particle feeder, which delivered particles of one predetermined diameter. The aperture plate collected most particles, allowing only an occasional particle to pass into the liquid and fall through the LDA measuring volume. The LDA was positioned $200 \mathrm{~mm}$ below the liquid surface: therefore, the particles were isolated and essentially at their terminal velocity condition when their velocity was measured.

Roughly 200 particle velocities were measured. Measurements yielded a mean terminal velocity of $0.05 \mathrm{~m} / \mathrm{s}$ with a standard deviation of $0.01 \mathrm{~m} / \mathrm{s}$. Analysis showed that the particle drag coefficient was generally 1.5 times larger than the standard drag correlation provided by [10]; therefore, $C_{D}$ was increased by this amount for all separated-flow calculations. A possible reason for the increased $C_{\mathrm{D}}$ was the irregular shape of some of the particles (roughly $60 \%$ were true spheres).

\subsection{Near-injector Properties}

Initial conditions for separated-flow calculations were measured at $x / d=8$, since high particle densities nearer to the injector tended to block LDA signals. The following measurements were made: $\bar{u}, \bar{u}^{\prime}, \bar{v}^{\prime}$ and $\overline{u^{\prime} v^{\prime}}$ for the liquid phase; $\tilde{u}_{\mathrm{p}}, \tilde{v}_{\mathrm{p}}, \tilde{u}_{\mathrm{p}}^{\prime}$ and $\tilde{v}_{\mathrm{p}}^{\prime}$ for the particles, where the Favre average denotes a particle-weighted average; and $\dot{n}^{\prime \prime}$ is the particle number flux. The symbols $\bar{u}^{\prime}$, $\bar{v}^{\prime}, \tilde{u}_{\mathrm{p}}^{\prime}$ and $\tilde{v}_{\mathrm{p}}^{\prime}$ represent root-mean-square fluctuating velocities.

The measurements at $x / d=8$ were supplemented by other estimates in order to complete the specification of initial conditions. The tangential components of the mean liquid and particle velocities were assumed to be zero, since the particles gave no indication of swirling motion. Tangential velocity fluctuations of both phases were assumed to be equal to their respective radial 


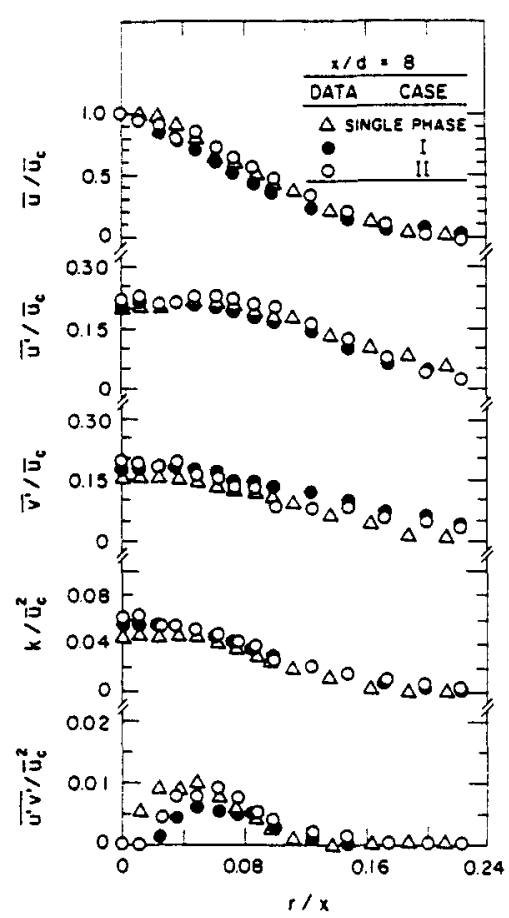

Figure 1. Mean and turbulent liquid properties at $x / d=8$.

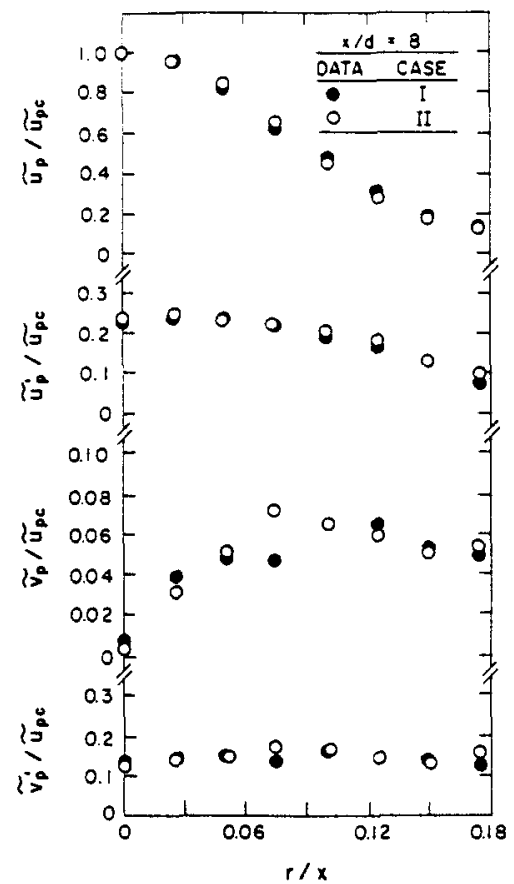

Figure 2. Mean and fluctuating particle velocities at $x / d=8$.

velocity fluctuations. This approximation provided initial values of $k$ for the liquid. Given the distributions of $\bar{u}, \overline{u^{\prime} v^{\prime}}$ and $k$, profiles of $\epsilon$ were estimated from the expression for the turbulent viscosity, [3], e.g.

$$
\epsilon=\frac{\mathrm{C}_{\mu} k^{2} \frac{\partial \bar{u}}{\partial r}}{\overline{u^{\prime} v^{\prime}}} .
$$

Liquid-phase properties for the single-phase and two particle-laden jets are illustrated in figure 1. Since the particle-laden jets were dilute, values of $\bar{u}, \bar{u}^{\prime}$ and $\bar{v}^{\prime}$ are not very different for the three flows. However, $k$ and $\overline{u^{\prime} v^{\prime}}$ are quadratic quantities which are more sensitive indicators of effects of particles. Near the axis, $\overline{u^{\prime} v^{\prime}}$ becomes smaller in the particle-laden jets, while velocity fluctuations and $k$ are increased. These effects tend to increase with increased particle loading and are evidence of turbulence modulation due to additional dissipation of the potential energy of particles.

Mean and fluctuating particle velocities at $x / d=8$ are illustrated in figure 2 . The properties of the two flows are essentially the same: $\tilde{u}_{\mathrm{p}}$ roughly parallels $\bar{u}$, while $\tilde{v}_{\mathrm{p}}$ increases near the axis and then remains relatively constant near the edge of the flow. Unlike mean radial liquid velocities (Wygnanski \& Fiedler 1969), there is no tendency for $\tilde{v}_{\mathrm{p}}$ to become negative near the edge of the flow, since particles are not entrained from the surroundings. Near the axis $\tilde{u}_{\mathrm{p}}^{\prime}>\tilde{v}_{\mathrm{p}}^{\prime}$, exhibiting greater anisotropy than fluctuating liquid velocities in this region (cf. figure 1). Similar to $\bar{u}^{\prime}$ and $\bar{v}^{\prime}$, however, $\tilde{u}_{\mathrm{p}}^{\prime}$ and $\tilde{v}_{\mathrm{p}}^{\prime}$ are comparable near the edge of the flow.

Particle number flux distibutions for the two particle-laden jets are illustrated in figure 3 . The distributions are reasonably symmetric about the axis. The half-widths of the distributions are roughly $r / x \sim 0.09$ and 0.10 for case I and II flows.

In contrast to the separated-flow calculations, initial conditions for the LHF approach were specified at the injector exit. The flow was assumed to be fully developed at the exit, with the distribution of $\bar{u}$ obtained from Schlichting (1979), and the distributions of $k$ and $\varepsilon$ obtained from Hinze (1975), all at the Reynolds number range of the present experiments.

\subsection{Properties Along the Axis}

Predicted and measured mean streamwise velocities along the axis, $\bar{u}_{\mathrm{c}}$, normalized by the jet exit velocity, $\bar{u}_{o}$, are illustrated in figure 4 . Results are shown for all methods of analysis - LHF, DSF, 


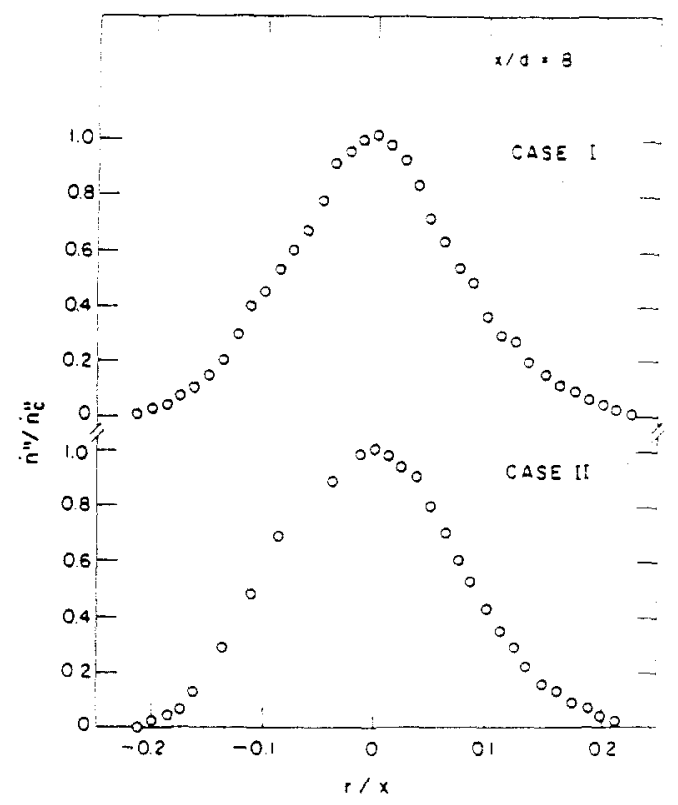

Figure 3. Mean particle number fluxes at $x / d=8$.

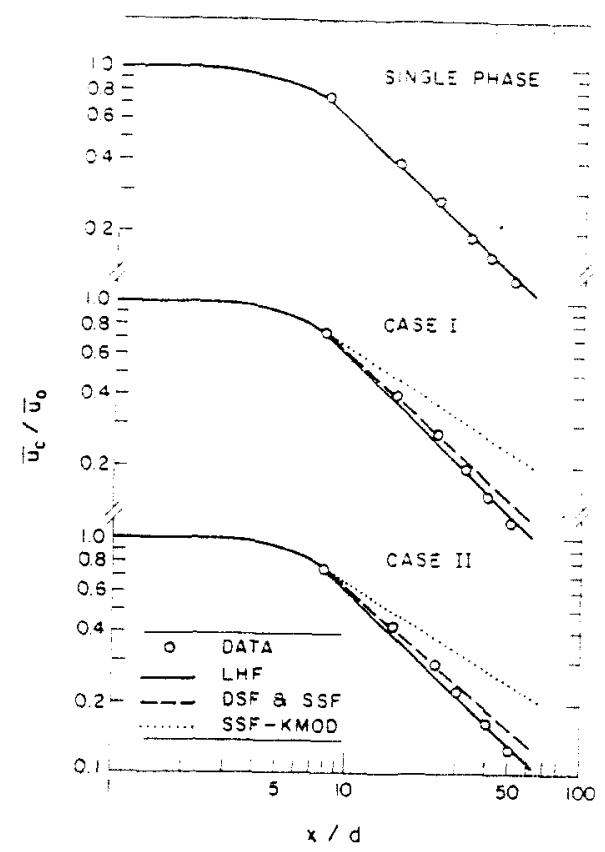

Figure 4. Mean liquid velocities along the axis.

SSF-BASE (the baseline version), SSF-KMOD (accounting for turbulence modulation in the $k$ equation) and SSF-EXT (accounting for turbulence modulation in both the $k$ and $\varepsilon$ equations).

Only LHF calculations, initiated at the jet exit, were carried out for the single-phase flow. Since the initial flow was fully-developed pipe flow, there is no potential core, although velocity changes along the axis are small for $x / d<3$. Farther downstream, centerline velocities decay according to $x^{-1}$, which is expected for single-phase round jets. The comparison between predictions and measurements is excellent-well within experimental uncertainties. This was true for other properties of the single-phase flow and these results will not be illustrated in the interest of brevity. These findings are typical of past experience concerning the performance of the present turbulence model for round jet flows (Jeng \& Faeth 1984; Shuen et al. 1985; Sun \& Faeth 1986), establishing a baseline for analysis of the particle-laden jets.

Mean liquid-phase velocities along the axis are illustrated for the two particle-laden jets in figure 4. The measurements are nearly identical to the single-phase jet, which is expected since the particle-laden flows were very dilute. The DSF, SSF-BASE and SSF-EXT predictions are the same and are represented by a single line for the particle-laden jet results illustrated in figure 4 . Except for the SSF-KMOD version, all methods of analysis agree reasonably well with each other and with the measurements. However, mean continuous-phase properties are not strongly influenced by the dispersed phase in dilute multiphase jets; therefore, this observation is a relatively weak indicator of the performance of these methods (Shuen et al. 1983a,b, 1985).

In contrast to the other methods illustrated in figure 4, the SSF-KMOD predictions substantially underestimate the rate of decay of $\bar{u}_{\mathrm{c}}$ with distance along the axis. This behavior is similar to the findings of Sun et al. (1986) and Reitz \& Diwakar (1987) for treatments of turbulence modulation limited to a source term in the governing equation for $k$. This term acts like a sink, reducing the turbulence kinetic energy of the flow, and thus, the rate of turbulent mixing. The problem appears to be an effect of scale, where the particles generally only influence the higher wavenumber end of the turbulence spectrum, rather than the large-scale turbulence which is primarily responsible for turbulent mixing (Hinze 1972). The SSF-EXT approach avoids the problem through the use of a particle source term in the governing equation for $\epsilon$, which apparently results in unchanged gross mixing levels in the present flows: The SSF-BASE method achieves the same objective (more crudely) by neglecting turbulence modulation entirely, which is tantamount to assuming that these phenomena occur on scales that do not influence the low wavenumber range of the turbulence spectrum which is responsible for mixing. This has been satisfactory for the particle-laden flows 


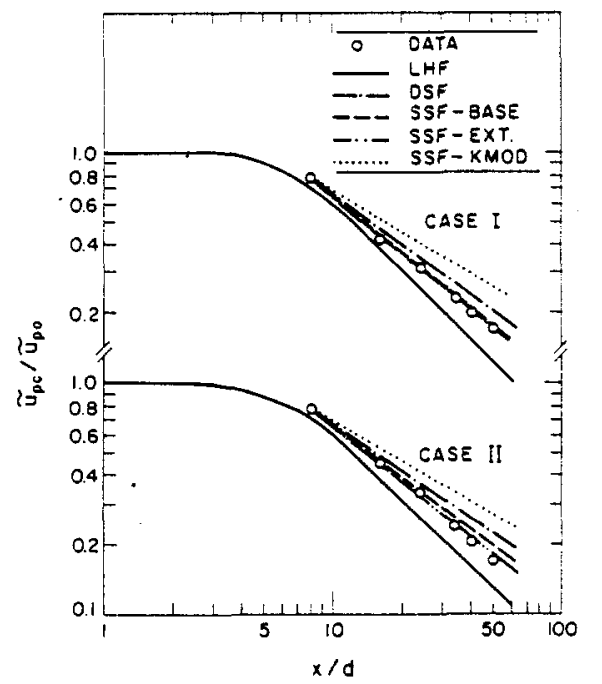

Figure 5. Mean particle velocities along the axis.

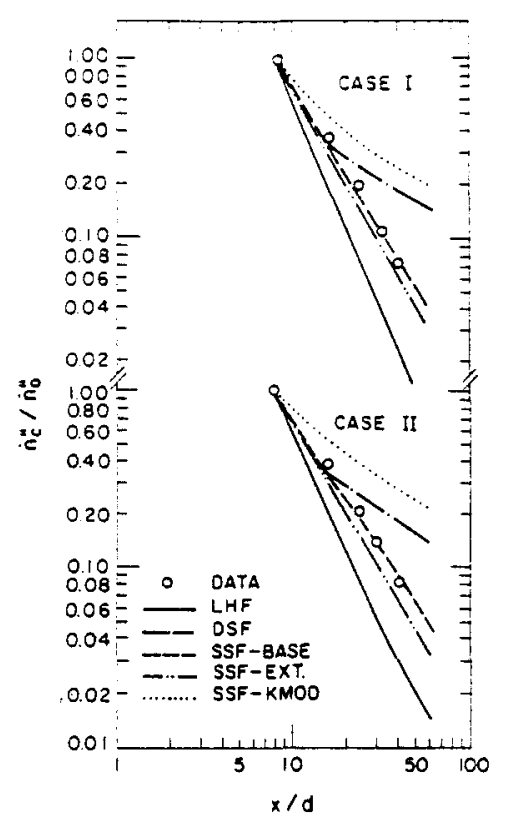

Figure 6. Mean particle number fluxes along the axis.

considered in this laboratory (Shuen et al. 1983a,b 1985; Sun \& Faeth 1986), but it is clearly not a reliable approach. Earlier studies by Al Taweel \& Landau (1977), Elghobashi \& Abou-Arab (1983) and others (cf. Faeth 1987), have sought to include the effects of scales on turbulence modulation. Based on the present findings, additional theoretical and experimental work along these lines is clearly needed.

The variation of streamwise mean particle velocities along the axis of the two particle-laden jets is illustrated in figure 5 . The measurements clearly show that mean particle velocities do not decay according to $x^{-1}$ like the liquid, which is the rate given by the LHF predictions. This behavior is caused by the finite inertia of the particles, which only separated-flow analysis can treat. As before, the SSF-BASE and SSF-EXT methods yield very similar predictions which are in reasonably good agreement with the measurements. Other separated-flow predictions are deficient to some extent. The SSF-KMOD approach underestimates the rate of decay of $\tilde{u}_{\mathrm{pc}}$, largely since it underestimates the rate of decay of $\bar{u}_{c}$. The DSF approach allows for particle inertia, but significantly underestimates the rate of decay of $\tilde{u}_{\mathrm{pc}}$, even though this approach yielded acceptable estimates of liquid velocities along the axis (cf. figure 4). This is an effect of the nonlinear drag law for the present conditions. Drag is almost a quadratic function of the relative velocity for the present conditions; therefore, when linearized, by using mean properties to represent the liquidphase properties, its magnitude is biased toward smaller values. This deficiency of DSF analysis has also been observed for nonevaporating sprays (Solomon et al. 1985a).

Predictions and measurements of particle number fluxes along the axis are illustrated in figure 6. The measurements roughly follow an $x^{-1}$ variation, rather than an $x^{-2}$ variation that would be expected for a passive contaminant in a round jet (which is similar to the LHF predictions illustrated in figure 6). This difference is due to the effects of particle inertia, which inhibits particles from diffusing like the fluid. Particle number fluxes along the axis are sensitive indicators of capabilities for predicting the turbulent dispersion of the particles; therefore, it is encouraging that the SSF-BASE and SSF-EXT methods yield predictions which are in reasonably good agreement with the measurements. The DSF method yields good results for a short distance after $x / d=8$, where initial conditions are specified. This is due to the imposition of measured mean particle radial velocities at the initial condition. Subsequently, the DSF method fails once these velocities decay in response to the mean liquid motion, due to neglect of particle/turbulence interactions responsible for turbulent dispersion. The SSF-KMOD results are even worse, due to poor predictions of liquid-phase mixing and turbulence levels-both of which reduce particle spread rates. 


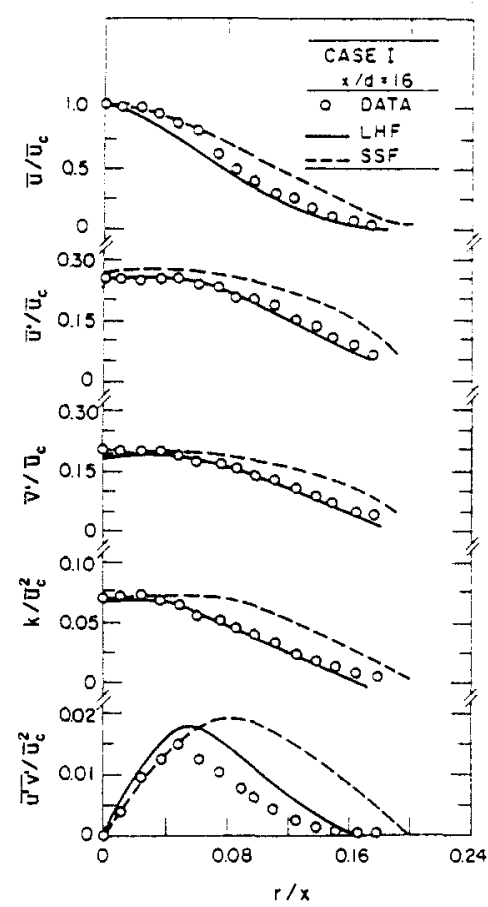

Figure 7. Mean and turbulent liquid properties (case I, $x: d=16$ )

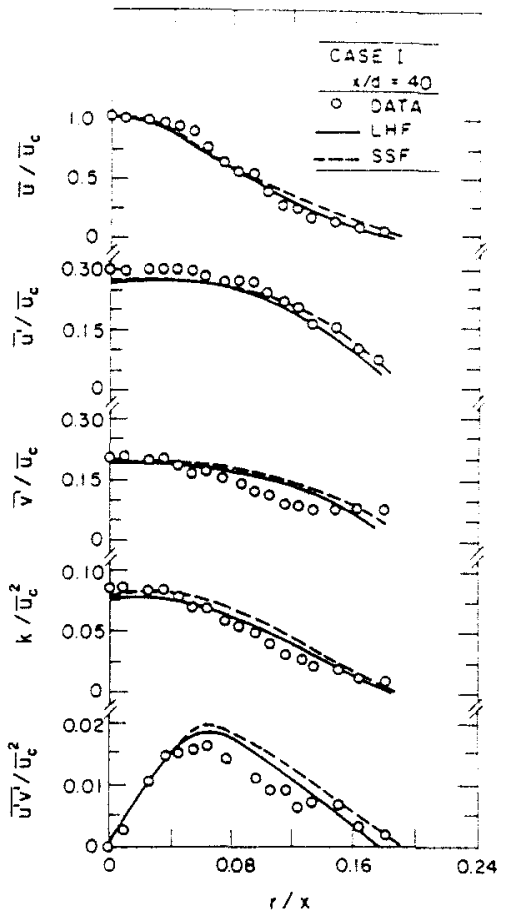

Figure 8. Mean and turbulent liquid properties (case I, $x / d=40)$.

\subsection{Liquid Properties}

Radial profiles of $\bar{u}, \bar{u}^{\prime}, \bar{v}^{\prime}$ and $\overline{u^{\prime} v^{\prime}}$ were measured at $x / d=16,24$ and 40; however, only the first and last positions will be considered in the following, since they are representative. Predictions of $\bar{u}^{\prime}$ and $\bar{v}^{\prime}$ were estimated from $k$, assuming $\bar{u}^{\prime 2}: \bar{v}^{\prime 2}=k: k / 2$, which are the ratios usually observed near the axis of single-phase jets (Wygnanski \& Fiedler 1969). Similar to results along the axis, measured values of $k$ were found by assuming $\bar{v}^{\prime}=\bar{w}^{\prime}$ (since $\bar{w}^{\prime}$ was not measured) which is also reasonable for jets (Wygnanski \& Fiedler 1969). In the following, flow variables will be plotted as a function of $r / x$, which is the similarity variable for fully-developed jets and plumes (Hinze 1975), in order to indicate estimates of flow widths.

Predictions and measurements of liquid properties for the two particle-laden jets at $x / d=16$ and 40 are illustrated in figures 7-10. LHF and SSF-BASE predictions are shown on the plots; however, SSF-EXT and DSF predictions were essentially identical to the latter. The LHF predictions appearing on the figures are essentially the same as results for the single-phase jet, due to the light particle loading of the present flows.

LHF and SSF predictions and the measurements at $x / d=40$, are all in reasonably good agreement (cf. figures 8 and 10). This performance is similar to earlier results for particle-laden jets in gases (Shuen et al. 1983a,b, 1985) and bubbles in liquids (Sun \& Faeth 1986). The LHF method also performs reasonably well at $x / d=16$; however, SSF predictions are not as satisfactory at this position. This problem is probably due to errors in the specification of initial conditions at $x / d=8$. The approach used to estimate $\epsilon$ is particularly problematical, since it involves several quadratic variables and the evaluation of a mean velocity gradient, leading to substantial uncertainties (the correctness of [3] aside). This view is supported by evaluation of the sensitivity of predictions to uncertainties in initial conditions, to be discussed later. In addition, LHF computations suggest relatively small effects of particles on liquid properties and yield better predictions, using initial conditions farther upstream, which provides more distance for errors in initial conditions to decay. Separated-flow predictions initiated at the injector exit (using estimated initial conditions), improve as well.

Another feature of the liquid-phase measurements is the progressive increase of $k$ (above predictions) near the axis as distance and initial particle loading are increased. This phenomenon 


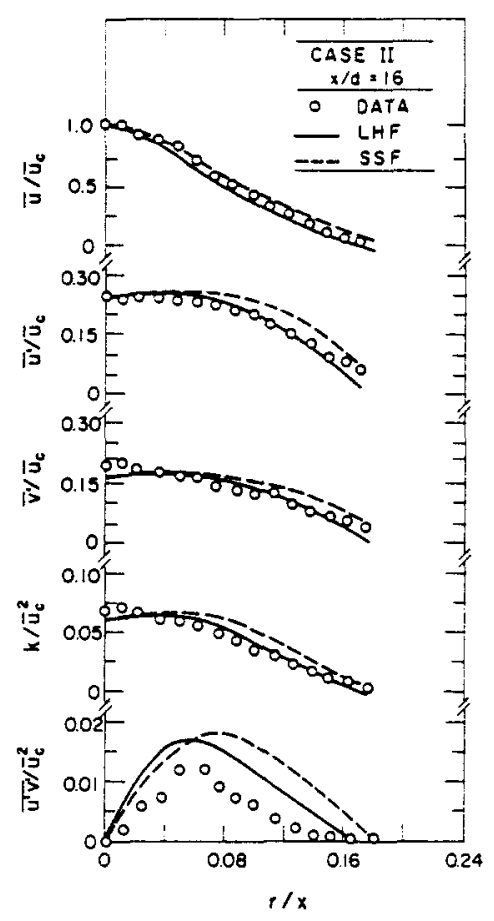

Figure 9. Mean and turbulent liquid properties (case II, $x / d=16$ )

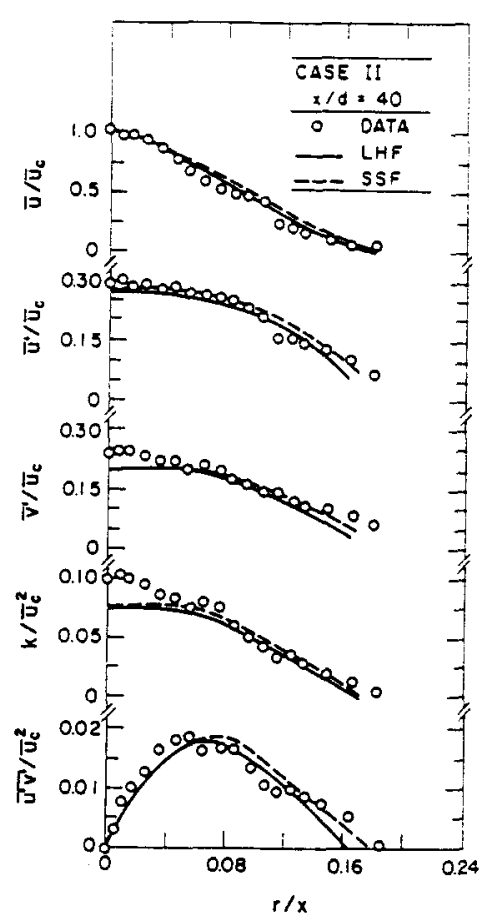

Figure 10. Mean and turbulent liquid properties (case II, $x / d=40$ ).

does not influence the gross mixing properties of the flow and is felt to be due to turbulence modulation. The effect on the turbulence spectra is probably limited to wavenumbers somewhat higher than the energy-containing range, but direct measurements are needed to assess this hypothesis. The effect is most evident near the axis, since conventional turbulence production by shear forces in the continuous phase is small in this region. The enhancement of $k$ grows with increasing distance from the injector, since liquid velocities are decreasing and becoming more comparable to velocity differences between the phases. Similar behavior has been observed in bubbly jets, far from the injector (Sun \& Faeth 1986; Sun et al. 1986). Additional analysis and measurements concerning turbulence modulation are clearly needed in order to gain a better understanding of this type of particle/turbulence interaction.

\subsection{Particle Velocities}

Predicted and measured mean and fluctuating particle velocities for the two particle-laden jets are illustrated in figures 11-14. Results are shown for $x / d=16$ and 40 . Since deficiencies of the DSF and SSF-KMOD approaches have already been discussed, predictions are only shown for the LHF, SSF-BASE and SSF-EXT methods. LHF predictions are virtually identical to predictions of liquid properties for all the methods.

The LHF method generally provides reasonably good predictions of particle properties, when normalized in the manner of figures 11-14. This is fortuitous, since the centerline velocities used to normalize the results are underestimated by the LHF approach, cf. figure 5 . The separated-flow predictions are in best agreement with unnormalized particle velocities.

Both separated-flow methods end to overestimate $\tilde{u}_{\mathrm{p}} / \tilde{u}_{\mathrm{pc}}$ at $x / d=16$, which is unusual since profiles of mean dispersed-phase velocities have generally been predicted reasonably well in the past (Shuen et al. 1983a,b, 1985; Sun \& Faeth 1986). However, this difficulty disappears at greater distances from the injector, cf figures 12 and 14 for $x / d=40$, suggesting that the discrepancy is probably due to deficiencies in specifying initial conditions at $x / d=8$.

Of the two separated-fiow analyses, the SSF-EXT version provides best agreement with measurements. This is not due to the inclusion of the effects of turbulence modulation, both SSF-BASE and SSF-EXT give very similar predictions of mean and fluctuating liquid-phase properties. The improvements are largely due to the consideration of anisotropic liquid-phase 


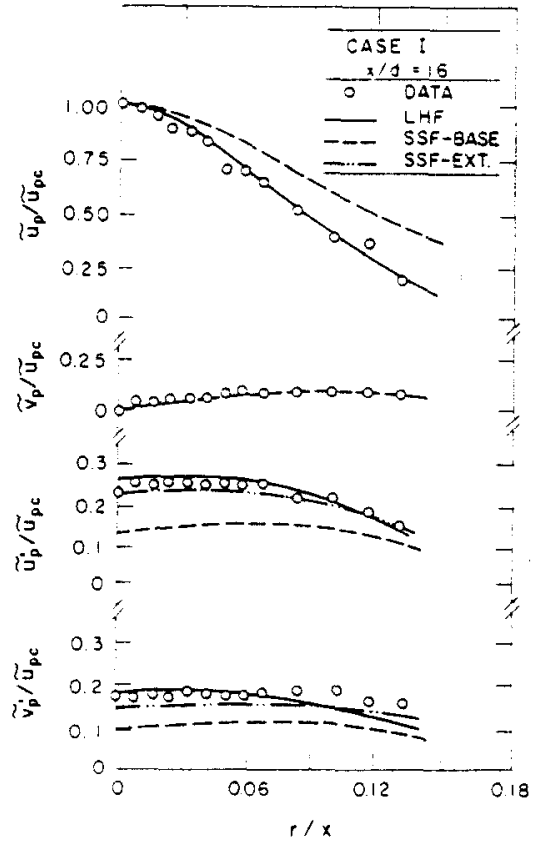

Figure 11. Mean and fluctuating particle velocities (case I, $x d=16$ ).

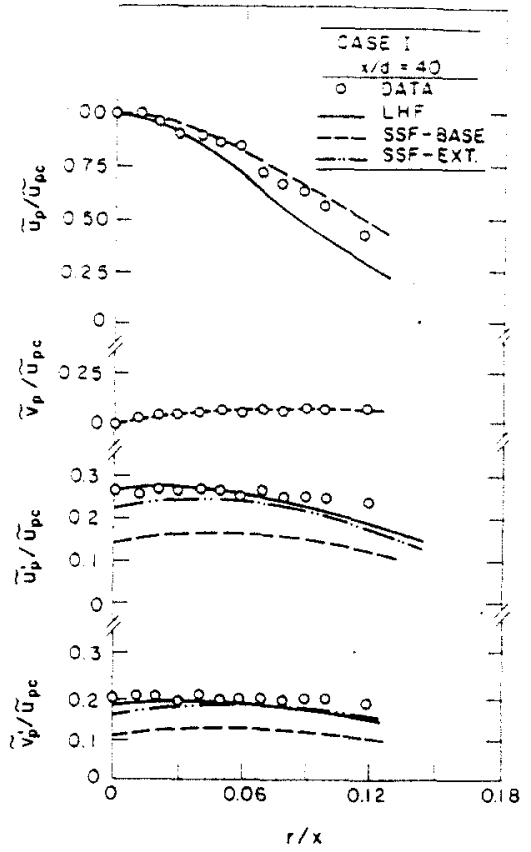

Figure 12. Mean and fluctuating particle velocities (case $I, x ; d=40$ )

velocity fluctuations and enhanced drag (resulting from large relative velocity fluctuations) by using [11] instead of [10] for particle drag predictions. For both particles in gases (Shuen et al. 1985) and bubbles in liquids (Sun et al. 1986), the assumption of isotropic velocity fluctuations for the continuous phase, during random-walk computations, was proposed as the reason for consistent underestimation of streamwise dispersed-phase velocity fluctuations. The present good predictions of both streamwise and radial particle velocity fluctuations, using the SSF-EXT method which allows for anisotropy of liquid velocity fluctuations, appears to confirm this hypothesis.

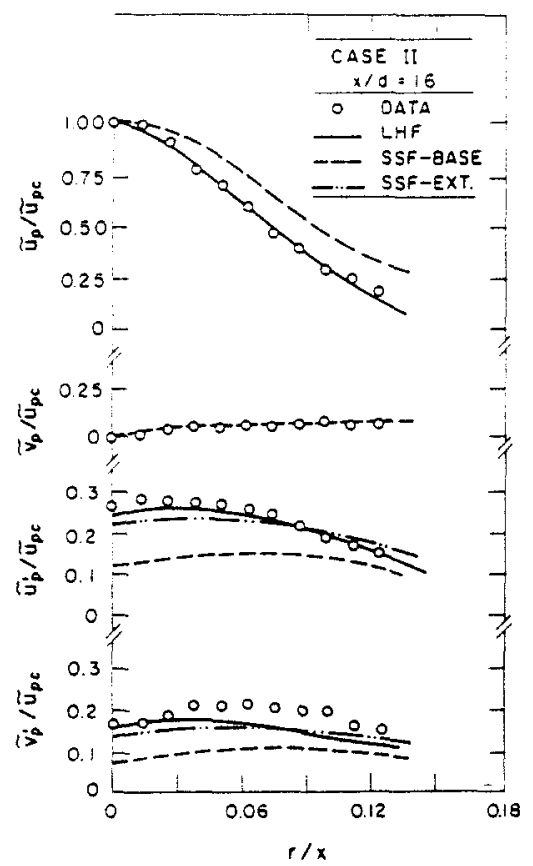

Figure 13. Mean and fluctuating particle velocities (case II, $x / d=16$ ).

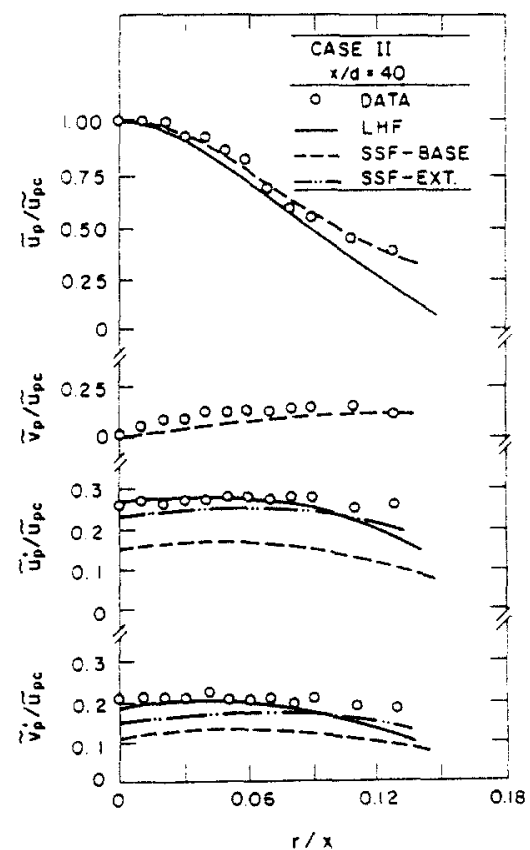

Figure 14. Mean and fiuctuating particle velocities (case II, $x / d=40$ ). 
The predictions of the SSF-BASE method in figures 11-14, not only underestimate the anisotropy of particle fluctuations, by ignoring the anisotropy of liquid velocity fluctuations, but underestimate radial velocity fluctuations as well. This has not been observed during past work with particles in gases and bubbles in liquids, using the SSF-BASE approach, where radial velocity fluctuations of the dispersed phase have been predicted reasonably well-consistent with reasonably good predictions of the turbulent dispersion of the dispersed phase (Shuen et al. 1985; Sun et al. 1986). This effect is probably due to the unusually high relative velocity fluctuations encountered by particles in liquids, in comparison to the other cases where terminal relative velocities of the dispersed phase were roughly an order of magnitude larger (reducing relative velocity fluctuations by a similar amount). A measure of the predicted effect of enhanced drag due to large relative velocity fluctuations can be seen by comparing predictions of $\tilde{v}_{\mathrm{p}}^{\prime} / \tilde{u}_{\mathrm{pc}}$ for the SSF-BASE and SSF-EXT methods, since the latter allows for the phenomenon. It is seen to be quite significant, roughly yielding a $40 \%$ increase in radial particle velocity fluctuations. However, the reasonably good results obtained here with the SSF-EXT method do not provide an adequate justification of the drag expression of [11]. The character of the continuous-phase turbulence spectrum and the size and response properties of the dispersed phase probably play a role in drag enhancement, and these properties are not considered in the drag correlation of [11]. Rather, the present results show that drag enhancement due to large relative velocity fluctuations can be important in particle-laden flows, and that this phenomenon deserves further study. Lopes \& Dukler (1986) also found enhanced drag effects, at high relative turbulence intensities, in annular two-phase flows.

\subsection{Particle Number Fluxes}

Predictions and measurements of particle number flux distributions, for the two particle-laden jets, are illustrated in figures 15 and 16 . Results are shown for $x / d=16,24$ and 40 . Predictions are presented for the LHF, DSF, SSF-BASE and SSF-EXT methods; however, the two separatedflow predictions were essentially the same and are simply denoted SSF in the figures.

Measurements on either side of the axis, illustrated in figures 15 and 16 , are reasonably symmetric, indicating good uniformity of particle distributions during the experiments. The LHF and SSF predictions are in good agreement wtih the measurements. However, the apparently good performance of the LHF method is an artifact of the method of plotting used in figures 15 and 16. Centerline concentrations, illustrated in figure 6, clearly show that the LHF approach

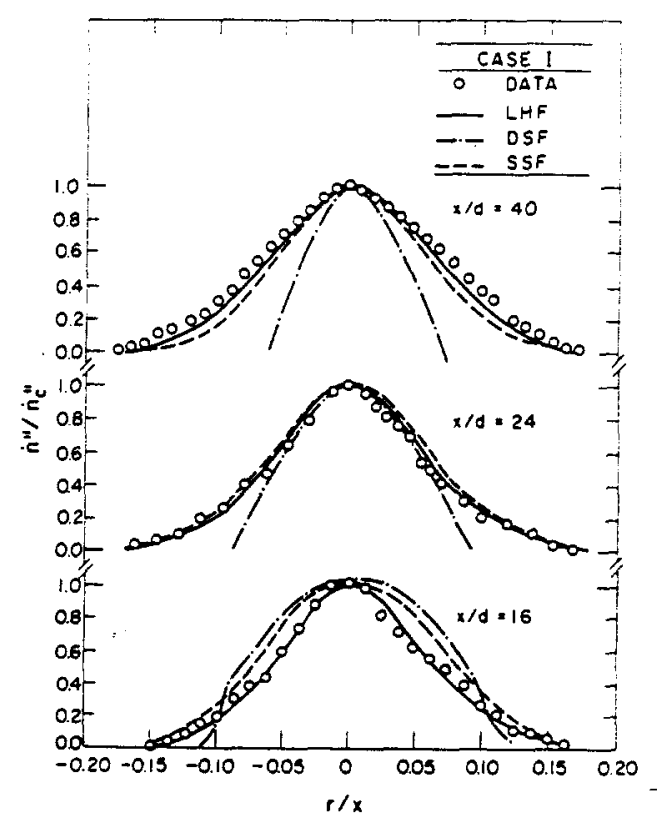

Figure 15. Mean particle number flux distributions (case 1).

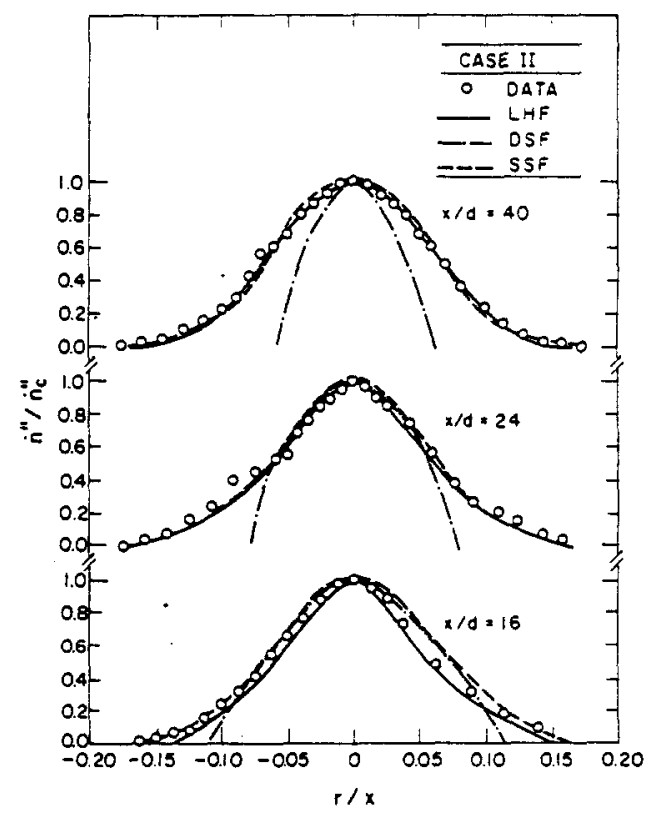

Figure 16. Mean particle number flux distributions (case II). 
Table 3. Sensitivity study of SSF analysis ${ }^{3}$

\begin{tabular}{lcccc}
\hline & \multicolumn{4}{c}{ Output parameter } \\
\cline { 2 - 5 } Input & $\tilde{u}_{s}$ & $k_{\mathrm{c}} / \bar{u}_{\mathrm{c}}^{2}$ & $\tilde{u}_{x}$ & $\tilde{u}_{\mathrm{x}} \tilde{u}_{x}$ \\
\hline Case $I$ & & & & \\
$k_{0}$ & $-36,-24$ & 219,24 & $-21,-25$ & 126,17 \\
$\epsilon_{0}$ & 24,17 & $-58,-8$ & 12,16 & $-27,-1$ \\
$C_{\mathrm{D}}$ & $\sim 0,-1$ & $-0,-2$ & $\sim 0,-3$ & 15,16 \\
$L_{\varepsilon}$ & $-1,-1$ & $1,-6$ & $-2,-4$ & 12,15 \\
Case II & & & & \\
$k_{0}$ & $-35,-23$ & 219,14 & $-20,-23$ & 117,98 \\
$\epsilon_{0}$ & 22,15 & $-56,-5$ & 16,15 & $-32,-4$ \\
$C_{\mathrm{D}}$ & $-1, \sim 0$ & $1,-4$ & $2,-3$ & $4,-1$ \\
$L_{c}$ & $-2,-2$ & $1,-7$ & $1,-2$ & 21,5 \\
\hline
\end{tabular}

Percentage increase in output parameter for a $100 \%$ increase of the input parameter at $x / d=16,40$.

overestimates the rate of turbulent dispersion of the particles, due to neglect of their inertia. Similarly, the DSF method is also unsatisfactory, due to neglect of particle/turbulence interactions. In contrast, the stochastic separated-flow methods yield resonably good results, consistent with their performance in figure 6. It appears that this approach can effectively treat effects of turbulent dispersion in jets for a wide range of conditions, e.g. the method has been reasonably successful for particles in gases, bubbles in liquids and now particles in liquids, which involves density ratios, $\rho_{\mathrm{p}} / \rho$, in the range $10^{-3}-10^{4}$.

\subsection{Sensitivity Study}

Evaluation of methods of predicting multiphase flows is always uncertain, since numerous theoretical and experimental parameters must be specified. Therefore, in order to place the evaluation of predictions in perspective, the sensitivity of predictions to changes in theoretical and experimental parameters was investigated. This involved increasing various parameters by $100 \%$ and finding changes in the dependent variables at $x / d=16$ and 40 for the two particle-laden jets.

Table 3 is a summary of the main results of the sensitivity analysis. Changes in $\bar{u}_{\mathrm{c}}, k_{\mathrm{c}} / \bar{u}_{\mathrm{c}}^{2}, \tilde{u}_{\mathrm{pc}}$ and $\tilde{u}_{\mathrm{pc}}^{\prime} / \tilde{u}_{\mathrm{pc}}$, the most sensitive output parameters, are tabulated for $100 \%$ increases in $k_{0}, \epsilon_{0}, C_{\mathrm{D}}$ and $L_{e}$. Errors in specification of initial conditions, $k_{0}$ and $\epsilon_{0}$, are very significant, particularly at $x / d=16$. This probably accounts for some of the deficiencies of SSF predictions noted at this position. Estimates of $C_{\mathrm{D}}$ have a small influence on liquid properties, consistent with the fact that present flows were dilute. $C_{\mathrm{D}}$ also has a relatively small effect on particle velocities, largely due to the fact that relative velocities are not large in the present flows, and drag is nearly a quadratic function of velocity. $C_{\mathrm{D}}$ has a much greater influence on particle velocity fluctuations, a finding already evident from the discussion of the results illustrated in figures 11-14. The parameter, $L_{\mathrm{e}}$, was specified in a relatively ad hoc manner (Shuen et al. 1983a), but has served resonably well for predictions of turbulent dispersion (Faeth 1987). Results summarized in table 3 indicate that most parameters of the flow are relatively insensitive to the actual value of $L_{e}$.

\section{CONCLUSIONS}

The major conclusions of the study are as follows:

(1) Stochastic separated-flow analyses yielded estimates of mixing and turbulent dispersion for particle-laden liquid jets that were comparable to past performance for particle-laden gas jets and bubbly jets-with no changes in the prescriptions for turbulence properties and turbulence/dispersed-phase interactions. Thus, the approach appears to be useful for a wide range of multiphase flows and merits further development.

(2) The effects of turbulence modulation were observed, evidenced by increased turbulence levels near the axis, where turbulence production by conventional continuous-phase mechanisms is small. The phenomenon did not appear to influence the overall mixing and turbulent dispersion properties of the flow, since 
the effects of particles on continuous-phase turbulence properties are probably limited to wavenumbers which are higher than the energy-containing range of the turbulence spectrum which is largely responsible for mixing. Several proposals for treating turbulence modulation were examined; however, none was particularly successful since they did not incorporate the effects of turbulence scale and the response of the dispersed phase. Additional measurements and analysis are clearly needed, in order to gain a better understanding of turbulence modulation in multiphase flows.

(3) The present flows exhibited higher relative turbulence intensities of the particles than in the past. This resulted in significant increases in particle drag from estimates based on the standard drag curve. An existing method, reported by Clift et al. (1978), exhibited some capacity to treat this phenomenon; however, since the method does not include effects of turbulence scale and particle response, its general use is suspect. Additional study of drag, virtual mass forces, Basset history forces and the effects of particle acceleration rates at high relative turbulence intensities is needed to gain a better understanding of this phenomenon.

(4) Earlier deficiencies in estimating the levels of anisotropy of dispersed-phase velocity fluctuations (Shuen et al. 1985; Sun \& Faeth 1986; Sun et al. 1986) were eliminated by considering measured levels of the anisotropy of continuous-phase velocity fluctuations. While this deficiency did not influence predictions of turbulent mixing and dispersion to a great degree for flows considered thus far, consideration of anisotropy of the continuous phase should be incorporated into stochastic separated-flow methods, since this may not always be the case.

(5) Predictably, the locally-homogeneous flow approximation yielded better results for the present particle-laden liquid jets, than for past work with particle-laden gas jets and bubbly jets, since relative velocities were smaller in comparison to continuous-phase velocities. Similar conditions are frequently encountered in high-pressure sprays, which are difficult to treat using the current separated-flow analyses; therefore, LHF methods merit further evaluation for such applications.

Acknowledgements - This research was supported by the Air Force Office of Scientific Research, Grant No. AFOSR-85-0244, under the Technical Management of J. Tishkoff. The authors also wish to acknowledge the assistance of T.-Y. Sun and J. P. Gore during the course of the work.

\section{REFERENCES}

Al Taweel, A. M. \& LandaU, J. 1977 Turbulence modulation in two-phase jets. Int. J. Multiphase Flow 3, 341-351.

BILgER, R. W. 1976 Turbulent jet diffusion flames. Prog. Energy Combust. Sci. 1, 87-109.

Clift, R., Grace, J. R. \& Weber, M. E. 1978 Bubbles, Drops and Particles, pp. 266-269, 296-302. Academic Press, New York.

DURST, F. \& WhITELAW, J. H. 1971 Measurements of mean velocity, fluctuating velocity and shear stress in air using a single channel optical anemometer. DISA Inf. 12, 11-16.

Elghobashi, S. E. \& ABOU-ARAB, T. W. 1983 A two-equation turbulence model for two-phase flows. Phys. Fluids 26, 931-938.

FAETH, G. M. 1983 The evaporation and combustion of sprays. Prog. Energy Combust. Sci. 9, 1-76.

FaETH, G. M. 1987 Mixing, transport and combustion in sprays. Prog. Energy Combust. Sci. In press.

GOSMAN, A. D. \& IOANNIDES, E. 1981 Aspects of computer simulation of liquid-fueled combustors. AIAA Paper No. 81-0323.

HINZE, J. O. 1972 Turbulent fluid and particle interaction. Progress in Heat and Mass Transfer, Vol. 6, pp. 433-452. Pergamon Press, Oxford.

HiNZE, J. O. 1975 Turbulence, 2nd edn, pp. 427, 724-734. McGraw-Hill, New York. 
JENG, S.-M. \& FAETH, G. M. 1984 Species concentrations and turbulence properties in buoyant methane diffusion flames. J. Heat Transfer 106, 721-727.

LockwOOD, F. C. \& NAGUIB, A. S. 1975 The prediction of fluctuations in the properties of free. round-jet turbulent diffusion flames. Combust Flame 24, 109-124.

LOPES J. C. \& DUKLER, A. E. 1986 Droplet dynamics in vertical gas-liquid flow. AlChE Jl. Submitted.

MODARRESS, D., TAN, H. \& Elghobashi, S. 1984 Two-component LDA measurements in a two-phase turbulent jet. AIAA $J l$ 23, 624-630.

ODAR, F.\& HAMILTON, W. S. 1964 Force on a sphere accelerating in a viscous fluid. J. Fluid Mech. $18,302-314$.

ReITz, R. D. \& DiwaKaR, R. 1987 Structure of high-pressure fuel sprays. SAE Paper 870598.

SCHLICHTING, H. 1979 Boundary Layer Theory, p. 599. McGraw-Hill, New York.

ShUEN, J.-S., ChEN, L.-D. \& FAETH, G. M. 1983a Evaluation of a stochastic model of particle dispersion in a turbulent round jet. AICHE $J / 29,167-170$.

Shuen, J.-S., Chen, L.-D. \& FAETH, G. M. 1983b Predictions of the structure of turbulent, particle-laden, round jets. $A I A A J l 21,1480-1483$.

SHUEN, J.-S., Solomon, A.S.P., ZhaNG, Q.-F. \& FAeTH, G. M. 1985 Structure of particle-laden jets: measurements and predictions. AIAA $J l$ 23, 396-404.

SHLEN, J.-S., SOlOMON, A. S. P. \& FAETH, G. M. 1986 Drop-turbulence interactions in a diffusion flame. AIAA $J l 24,101-108$.

Solomon, A. S. P., SHUEN, J.-S., ZHANG, Q.-F. \& FAETH, G. M. 1985a Structure of nonevaporating sprays: I. Near-injector conditions and mean properties. AIAA Jl 23, 1548-1555. ibid., II. Drop and turbulence properties. AIAA $J l 23,1724-1730$.

Solomon, A. S. P., Shuen, J.-S., Zhang, Q.-F. \& Faeth, G. M. 1985 b Measurements and predictions of the structure of evaporating sprays. J. Heat Transfer 107, 679-686.

SPALDING, D. B. 1977 GENMIX: a General Computer Program for Two-dimensional Parabolic Phenomena. Pergamon Press, Oxford.

SuN, T.-Y. \& FAETH, G. M. 1986 Structure of turbulent bubbly jets-I. Methods and centerline properties; -II. Phase property profiles. Int. J. Multiphase Flow 12, 99-126.

Sun, T.-Y., Parthasarathy, R. N. \& FaETH, G. M. 1986 Structure of bubbly round condensing jets. J. Heat Transfer 108, 951-959.

WYGNANSKI, I. \& FIEDLER, H. E. 1969 Some measurements in the self-preserving jet. J. Fluid Mech. 38, 577-612. 